\title{
Resistance is not futile: gliotoxin biosynthesis, functionality and utility
}

\author{
Stephen K. Dolan", Grainne O'Keeffe ${ }^{*}$, Gary W. Jones, and Sean Doyle
}

Department of Biology, Maynooth University, Maynooth, County Kildare, Ireland

\begin{abstract}
Gliotoxin biosynthesis is encoded by the gli gene cluster in Aspergillus fumigatus. The biosynthesis of gliotoxin is influenced by a suite of transcriptionally-active regulatory proteins and a bis-thiomethyltransferase. A selfprotection system against gliotoxin is present in $A$. fumigatus. Several additional metabolites are also produced via the gliotoxin biosynthetic pathway. Moreover, the biosynthesis of unrelated natural products appears to be influenced either by gliotoxin or by the activity of specific reactions within the biosynthetic pathway. The activity of gliotoxin against animal cells and fungi, often mediated by interference with redox homeostasis or protein modification, is revealing new metabolic interactions within eukaryotic systems. Nature has provided a most useful natural product with which to reveal some of its many molecular secrets.
\end{abstract}

\section{Contextualizing and rethinking gliotoxin}

Aspergillus fumigatus is an opportunistic fungal pathogen and primarily infects immunocompromised individuals where it can cause fatal invasive aspergillosis (IA) [1]. A. fumigatus exposure can also induce debilitating aspergillosis and allergy in immunocompetent individuals [2,3]. Selected secondary metabolites produced by A. fumigatus, in particular siderophores and the non-ribosomal peptide gliotoxin, are generally considered to be front-line virulence factors $[4,5]$. Gliotoxin is an epipolythiodioxopiperazine (ETP) of molecular mass $326 \mathrm{Da}$, and contains a disulfide bridge which can undergo repeating cleavage and reformation, thereby resulting in a potent intracellular redox activity (Figure 1) [6]. Indeed, the dithiol form of gliotoxin has also been posited to be responsible for the observed biological activities of gliotoxin [7]. Bisdethiobis(methylthio)gliotoxin (BmGT) and related gliotoxin metabolites (Figure 1) are also biosynthesized by A. fumigatus $[8,9]$. Incredibly, the gliotoxin biosynthetic pathway had remained elusive since the discovery of gliotoxin in 1936; however, recent studies have not only dissected this unusual molecular assembly system but have revealed the necessity for gliotoxin-producing fungi to possess an endogenous resistance system against gliotoxin $[10,11]$. Moreover, because gliotoxin can be considered as

Corresponding author: Doyle, S. (sean.doyle@nuim.ie).

Keywords: Aspergillus fumigatus; NRPS; natural products; secondary metabolism; antioxidant.

"These authors contributed equally to this work.

0966-842X/

(C) 2015 Elsevier Ltd. All rights reserved. http://dx.doi.org/10.1016/j.tim.2015.02.005 the prototype ETP, studies on gliotoxin can be instrumental in revealing the biosynthetic mechanisms of related ETPs which are biosynthesized by a range of fungi [12]. Amongst others, these include sirodesmin A, sporidesmin A, chaetocin, aranotin, and chetomin [13]. Studies on the biosynthetic mechanism of ETPs, particularly gliotoxin, are also serving to inspire new synthetic chemistry approaches for ETP synthesis and desulfurization, which are somewhat beyond the scope of the present review $[13,14]$.

In addition to studying how gliotoxin contributes to organismal virulence, it has also been deployed to explore and reveal novel biochemistry within both fungal and animal cells $[15,16]$. Thus, we contend that it is the ability of gliotoxin to interfere with so many cellular processes that makes it such a useful tool to access the many important, although occluded, systems interactions within fungi. Indeed, emerging studies of the reciprocal effects of gliotoxin on animal cells and fungi are serving to enhance our view of gliotoxin as an antioxidant as much as a toxin $[6,17]$. In this review we set out our current understanding of gliotoxin biosynthesis, its regulation, and resistance systems against gliotoxin. We also attempt to elucidate how the effects of gliotoxin on fungi and animal cells can cross-fertilize our knowledge of hitherto unknown aspects of both eukaryotic cell systems.

\section{Gliotoxin biosynthesis}

The in silico identification of the gli cluster in A. fumigatus (Figure 1) [18] paved the way for multiple functional genomic studies which revealed that the gli cluster, comprising 13 genes, encodes the key enzymes responsible for gliotoxin biosynthesis (Figure 2). The $\mathrm{Zn}(\mathrm{II})_{2}$-Cys(6) binuclear cluster domain transcription factor gliZ was confirmed as the regulator of the gli cluster [19], whereby deletion of this gene abrogated the expression of key biosynthetic genes and concomitant gliotoxin biosynthesis. Interestingly, upon gliZ reconstitution in A. fumigatus $\Delta g l i Z$, the biosynthesis of unrelated natural products was induced. The non-ribosomal peptide synthetase, GliP, catalyzes the formation of cyclo-phenylalanyl-serine, the first biosynthetic intermediate in the gliotoxin biosynthetic pathway [20], and multiple groups undertook gliP deletion, which results in complete loss of gliotoxin biosynthesis [5,21-23] (Figure 2). Interestingly, Cramer et al. observed that exogenous gliotoxin induced gli cluster gene expression in A. fumigatus, which was somewhat attenuated in A. fumigatus $\Delta g l i P$ [21]. Although not unheard of, it is certainly unusual for a fungal natural product to induce its own biosynthesis, and the biological significance of this 


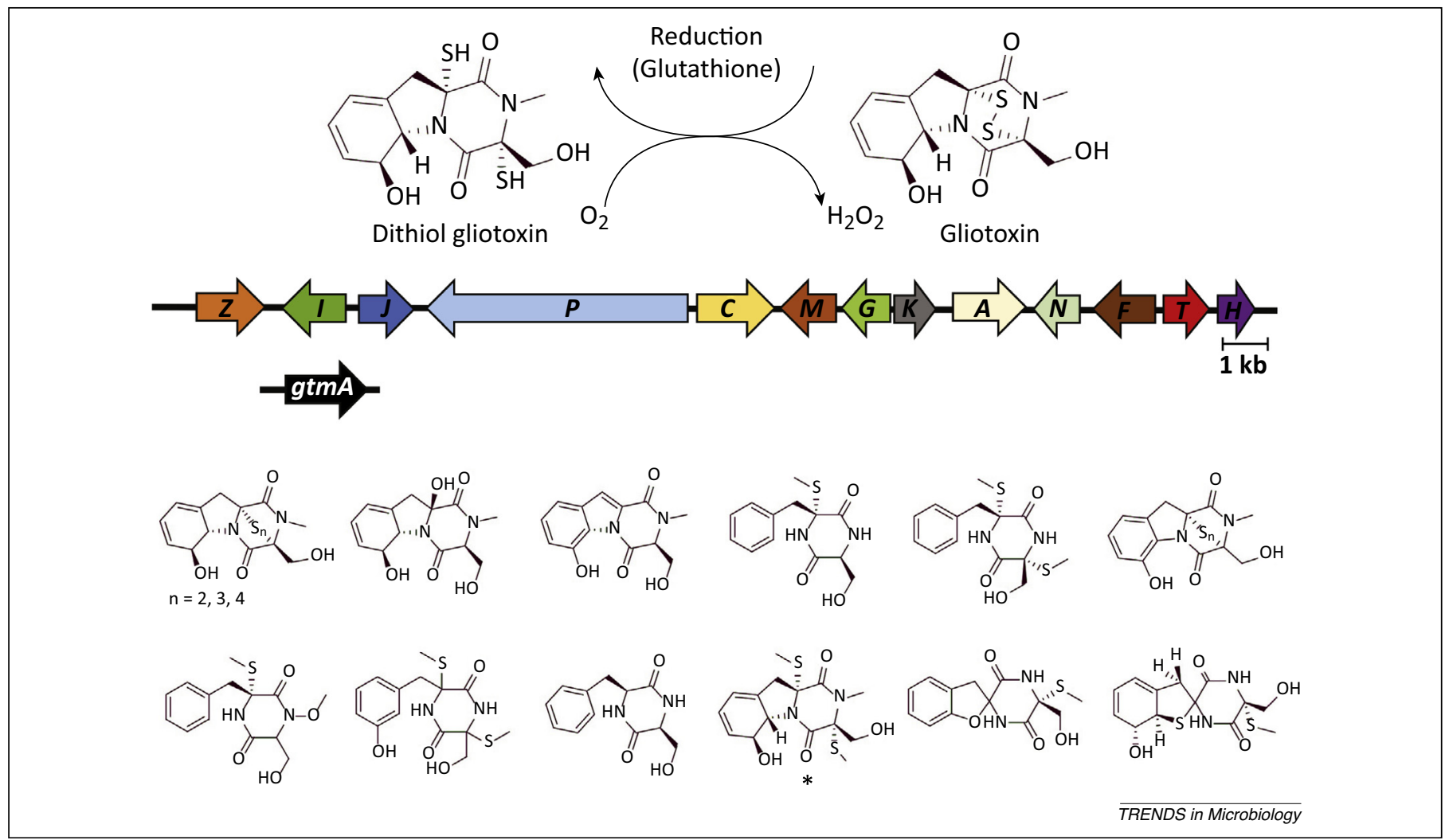

Figure 1. Interconversion of gliotoxin between the reduced (dithiol) and oxidized (disulfide) forms. In Aspergillus fumigatus the gli cluster, which encodes gliotoxin biosynthesis and consists of 13 genes (in color and labeled with their last letter), is located on chromosome 6 [10,18]. gtmA is encoded outside the cluster and is on

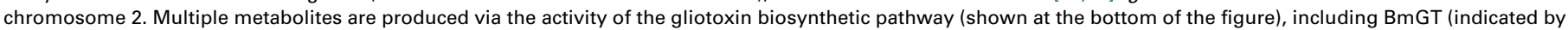
an asterisk) [9].

phenomenon is only beginning to emerge. Cyclophenylalanyl-serine is subsequently converted to a highly-reactive acyl imine intermediate before bis-glutathionylation. GliC has been shown to catalyze hydroxylation of the $\alpha$-carbon of L-Phe in cyclo-phenylalanyl-serine before bis-glutathionylation [24]. The demonstration that gliG encodes a glutathione $S$-transferase (GST), which conjugates two glutathione (GSH) molecules to a biosynthetic intermediate to form a bis-glutathionylated biosynthetic intermediate (Figure 2), and which is therefore responsible for the sulfurization of gliotoxin, was an important finding because it was one of the first demonstrations that GSTs played a biosynthetic, as opposed to detoxification, role in fungi $[25,26]$. This bis-glutathionylated intermediate is subsequently processed by GliK, a $\gamma$-glutamyl cyclotransferase, to remove both $\gamma$-glutamyl moieties $[17,27]$. Subsequent processing via GliI [28] yields a biosynthetic intermediate, which is $N$-methylated via the $N$-methyltransferase GliN, before the gliotoxin oxidoreductase GliT-mediated disulfide bridge closure (Figure 2) [27,29]. Gliotoxin secretion from A. fumigatus ensues, now known to be facilitated in part by the MFS transporter GliA [30].

\section{Regulation of gliotoxin biosynthesis}

Dolan et al. have identified a negative regulatory mechanism which deploys a non-gli cluster encoded gliotoxin bisthiomethyltransferase, termed GtmA [8]. gtmA expression is induced by gliotoxin, and GtmA (also termed TtmA in [29]) functions to bis-thiomethylate dithiol gliotoxin to form BmGT [8,29], instead of undergoing disulfide bridge closure via GliT (Figure 2). Consequently, both gliotoxin biosynthesis and auto-induction of the gli cluster are attenuated. Importantly, this post-biosynthetic negative regulatory mechanism may be operative in other fungal ETP biosynthetic systems because bis-thiomethylated ETPs are produced by fungi, and GtmA orthologs have also been identified across the ascomycetes [8,29]. Moreover, overproduction of gliotoxin or related ETPs for biotechnological purposes may be dependent on utilizing bis-thiomethyltransferase-deficient strains to deactivate this negative feedback system. Although GliH has been shown to be essential for gliotoxin biosynthesis [10], to date the function of this enzyme has not been elucidated.

Interestingly, apart from gliZ, many other transcription factors and regulatory genes influence gliotoxin biosynthesis (Table 1). The $\mathrm{C}_{2} \mathrm{H}_{2}$ transcription factor gip $A$ regulates gliA interdependently with gliZ [31]. In addition, it is wellestablished that laeA, a global regulator of secondary metabolism, which interacts with the velvet proteins VeA and VelB in the nucleus [32-35], augments gliotoxin biosynthesis because either laeA or veA deletion results in impaired gliotoxin production in A. fumigatus [34,36]. Other transcription factors found to regulate the gli cluster include $m t f A$ (a $\mathrm{C}_{2} \mathrm{H}_{2}$ transcription factor [37]), rsmA (a bZIP transcription factor [38]), and stuA (an APSES family transcription factor $[39,40]$ ) (Table 1 ). The developmental regulators $f l b B$ and $f l b E$ positively influence gliotoxin biosynthesis because deletion mutants exhibit reduced or abolished gliotoxin production [41-43]. The 
<smiles>N[C@@H](Cc1ccccc1)C(=O)OCC(=O)O</smiles>

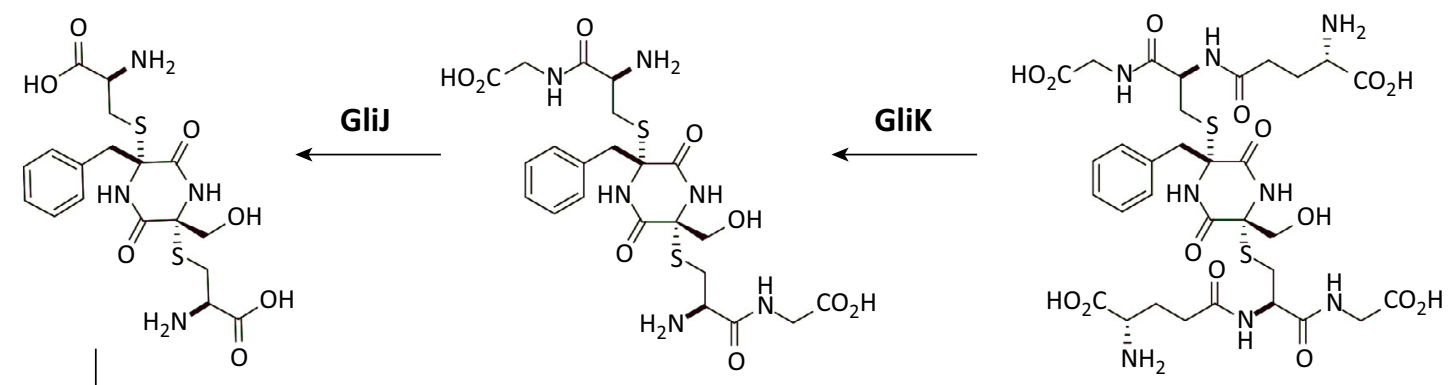

Glil<smiles>C[C@H](CO)[C@H]1NC(=O)[C@H](CO)NC1=O</smiles>

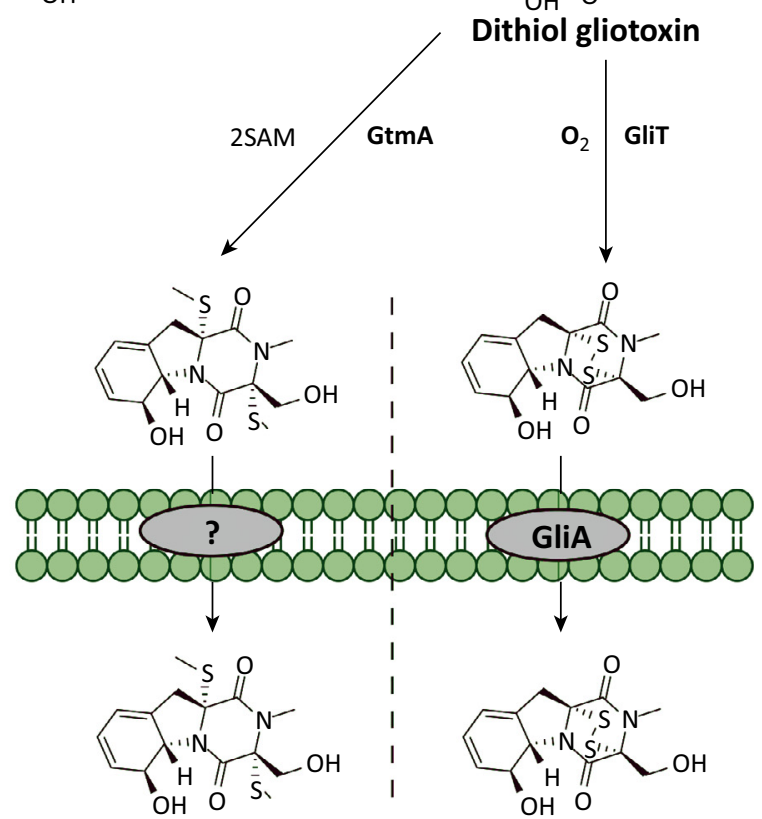

Bisdethiobis(methylthio)gliotoxin

Gliotoxin

$\overline{\text { TRENDS in Microbiology }}$

Figure 2. Gliotoxin biosynthesis, secretion, and regulation. A series of enzyme-catalyzed steps convert phenylalanine and serine to gliotoxin or BmGT via the formation of reactive intermediates, bis-glutathionylation, degradation steps, methylation, and oxidation $[10,11,15,20,25,26]$. Dithiol gliotoxin, a co-substrate for either GliT or GtmA, is converted to gliotoxin or BmGT, respectively [8,83]. Gliotoxin can induce gli cluster expression [21] or is secreted from A. fumigatus via GliA [30]; however, the process of $\mathrm{BmGT}$ release is undefined. BmGT formation and secretion dissipate intracellular gliotoxin, thereby preventing gli cluster expression leading to downregulation of gliotoxin biosynthesis [8]. Please note that the precise order of some reaction steps, and gliM/H involvement, remain to be fully elucidated.

MAP kinase, $m p k A$, and the class 2 histone deacetylase, $h d a A$, also positively regulate gliotoxin biosynthesis while the histone methyltransferase, $c c l A$, negatively regulates gliotoxin production because increased gliotoxin levels were evident in $\Delta c c l A$, consequent to increased gliZ expression [44] (Table 1). Thus, it is now apparent that regulation of gliotoxin biosynthesis is a hierarchical, multifactorial process that is affected by many regulatory elements, and which is highly integrated into A. fumigatus metabolism. 
Table 1. Transcriptional regulators which impact upon gli cluster gene expression and gliotoxin biosynthesis

Transcriptional regulator

GliZ

$\left(\mathrm{Zn}_{2} \mathrm{Cys}_{6}\right.$ binuclear

transcription factor)

GipA

$\left(\mathrm{C}_{2} \mathrm{H}_{2}\right.$ transcription factor)

LaeA

(transcriptional regulator)

VeA

(regulatory gene)

FlbA

(regulator of G-protein signaling protein)

MpkA

(MAP kinase)

\section{MtfA}

$\left(\mathrm{C}_{2} \mathrm{H}_{2}\right.$ transcription factor)

RsmA

(restorer of secondary metabolism - A bZIP

transcription factor)

CclA

(histone

methyltransferase)

\section{Effect on gliotoxin biosynthesis ${ }^{a}$}

Essential for gliotoxin biosynthesis. Deletion results in loss of expression of some of the other gli cluster genes (e.g., glil, gliA, gliG).

Regulates gliotoxin biosynthesis genes; decreased expression of gliA, gliZ, gliP, and gliT, and consequently reduced gliotoxin production in $\Delta$ gipA. Conversely, these genes had increased expression in OEgipA.

The gligenes (except gliM) contain potential GipA binding sites. GipA cannot induce gliA or gliP expression independently of $g / i Z$, while $g / i Z$ induction of gliA is GipA-dependent.

Positively regulates gliotoxin biosynthesis because gliotoxin production is impaired in $\Delta / a e A$.

Positively regulates gliotoxin biosynthesis because reduced gliotoxin production is evident in $\Delta v e A$. gliZ and gliPexpression are decreased in $\Delta v e A$.

Does not affect gliotoxin biosynthesis; however, expression of gliT and secretion of GliT are increased in $\triangle \mathrm{fl} / \mathrm{bA}$. Consequently, $\triangle \mathrm{flb}$ A displays enhanced tolerance to exogenous gliotoxin.

Regulates gliotoxin production which is reduced in $\triangle m p k A$ compared to wild type. Expression of gliN and gliT is decreased in $\triangle m p k A$.

Gliotoxin levels were increased in OEmtfA but were unchanged in $\triangle m t f A$. Expression of $g l i Z$ and gliP was increased in OEmtfA.

The production of 12 gli cluster metabolites was increased 2-100-fold in the OErsmA strain compared to wild type.

Increased gliotoxin production in $\Delta c c / A$ mediated by a substantial increase in gliZ transcription.

\section{Other phenotypes}

gliZ regulates the production of other SM (e.g.,

OEgliZ produced helvolic acid at $37^{\circ} \mathrm{C}$, unlike wild type). Virulence in a mouse model is not significantly different from wild type, although is slightly decreased. Involved in apoptotic but not necrotic cell death.

GipA potentially induces other SM clusters because at least one gene from 18 SM clusters was increased in expression in an OEgipA strain.

Global regulator of SM production in $A$.

fumigatus because 13 of $22 \mathrm{SM}$ clusters exhibited reduced expression in $\Delta / a e A$. $\Delta / a e A$ had reduced virulence in a mouse model of IA. $\Delta / a e A$ conidia were defective in rodlet production and had increased susceptibility to macrophage phagocytosis, while hyphae displayed decreased ability to kill neutrophils. $\triangle / a e A$ demonstrated impaired conidia and conidiophore production in liquid shake culture.

VeA predominantly positively regulates other SM clusters; fumagillin, fumitremorgin G,

fumigaclavine $\mathrm{C}$, and glionitrin $\mathrm{A}$ production is reduced in $\triangle v e A$. VeA regulates development; $\Delta v e A$ demonstrates reduced conidiation but increased pigmentation of conidia, reduced protease activity, and increased expression of brlA (a developmental activator) during development compared to wild type. VeA is dispensable for virulence in a neutropenic mouse infection model. VeA is highly conserved; $v e A$ from Aspergillus nidulans complements deletion in A. fumigatus.

laeA expression is reduced in $\triangle \mathrm{flbA}$, suggesting that other SM clusters may be effected by FlbA. $\mathrm{FlbA}$ is necessary for normal cell death progression and autolysis in submerged cultures. $\triangle f l b A$ has increased superoxide dismutase and catalase activity, and demonstrated enhanced resistance to menadione and paraquat. FlbA downregulates hyphal differentiation by inactivating $\mathrm{GpaA}$, and thus induces asexual development.

MpkA is activated by iron starvation, to which it responds by reshuffling the amino acid pool (increased ornithine levels) leading to increased siderophore biosynthesis. $\triangle m p k A$ exhibits increased sensitivity to menadione and diamide but decreased sensitivity to $\mathrm{H}_{2} \mathrm{O}_{2}$. Involved in cell wall integrity signaling.

Involved in growth and development; reduced growth and conidiation was observed in $\triangle m t f A$ and OEmtfA.

Positive regulator of protease production. $\triangle m t f A$ exhibited attenuated virulence in Galleria mellonella.

Involved in growth and oxidative stress resistance; OErsmA exhibits a growth defect at low growth temperature $\left(25^{\circ} \mathrm{C}\right)$. OE rsmA mutants show increased resistance to menadione.

Increased production of other unidentified secondary metabolites. Severe growth defects and sensitivity to 6 -azauracil (6AU); an inhibitor of guanine nucleotide synthesis and an indicator of transcriptional defects. 
Table 1 (Continued)

\begin{tabular}{|c|c|c|c|}
\hline Transcriptional regulator & Effect on gliotoxin biosynthesis ${ }^{a}$ & Other phenotypes & Refs \\
\hline $\begin{array}{l}\text { FlbB } \\
\text { [developmental regulator } \\
\text { containing a basic leucine- } \\
\text { zipper domain (bZIP)] }\end{array}$ & $\begin{array}{l}\text { Drastically reduced gliotoxin production ( } 85 \%) \\
\text { in the } \Delta f l b B \text { mutant compared to wild type. }\end{array}$ & $\begin{array}{l}\text { Delayed/reduced sporulation; precocious cell } \\
\text { death; lack of conidiophore development in } \\
\text { submerged culture, reduced ( } \sim 90 \%) \text { fumagillin } \\
\text { production. }\end{array}$ & {$[42,90]$} \\
\hline $\begin{array}{l}\text { FlbE } \\
\text { (developmental regulator } \\
\text { interacts with FlbB) }\end{array}$ & $\begin{array}{l}\text { Gliotoxin production was abolished in the } \Delta f l b E \\
\text { deletion strain. }\end{array}$ & $\begin{array}{l}\text { Controls asexual development: deletion results in } \\
\text { reduced conidiation and delayed expression of } \\
b r l A \text { and vosA. Necessary for salt-induced } \\
\text { development in liquid submerged culture. }\end{array}$ & {$[43,90]$} \\
\hline $\begin{array}{l}\text { HdaA } \\
\text { (class } 2 \text { histone } \\
\text { deacetylase) }\end{array}$ & $\begin{array}{l}\text { Deletion of the } h d a A \text { gene decreased gliotoxin } \\
\text { production and overexpression of } h d a A \\
\text { increased gliotoxin production. }\end{array}$ & $\begin{array}{l}\Delta h d a A \text { demonstrated a reduction in both } \\
\text { germination rate and vegetative growth; altered } \\
\text { colony morphology and increased expression of } \\
\text { several NRPS genes. }\end{array}$ & {$[50]$} \\
\hline $\begin{array}{l}\text { StuA } \\
\text { (APSES family } \\
\text { transcription factor) }\end{array}$ & $\begin{array}{l}\text { gliP expression, and likely gliotoxin production, } \\
\text { are dependent on StuA in vivo. }\end{array}$ & $\begin{array}{l}\Delta \text { stuA had severely impaired conidiation; six } \\
\text { secondary metabolite clusters were found to be } \\
\text { regulated by StuA. }\end{array}$ & {$[39,40]$} \\
\hline
\end{tabular}

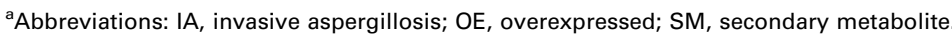

\section{Histone H3 lysine 9 (H3K9) methyltransferase inhibition} Gliotoxin, and indeed other ETPs (e.g., chetomin and chaetocin), are inhibitors of H3K9 methyltransferases, which has been demonstrated using recombinant enzymes $[45,46]$. The disulfide bond of ETP molecules is crucial for this inhibition because ETPs in which the thiols are rendered unreactive (e.g., bis-dethiobisacetylgliotoxin and chaetocin with methylated sulfur groups) do not display inhibitory abilities $[45,46]$. While direct investigations of the effects of gliotoxin on histone H3K9 methyltransferases in A. fumigatus or other filamentous fungi have not been performed to the best of our knowledge, functional studies of histone-modifying enzymes have been carried out (reviewed in [47]). Disruption of histone modifications, in particular methylation and acetylation of histone $\mathrm{H} 3$ tail residues, has been linked with alterations in not only gliotoxin production but also in secondary metabolite production in general in the Aspergilli [47-49]. A member of the conserved eukaryotic H3K4 methylating COMPASS complex, CclA, is required for tri- and di-methylation of H3K4 and regulates secondary metabolite production [44,48]. Deletion of $c c l A$ resulted in increased production of gliotoxin and other secondary metabolites, leading to the conclusion that tri- and di-methylation of H3K4 play a part in secondary metabolite production in A. fumigatus [44]. In addition, the class 2 histone deacetylase, HdaA, also regulates secondary metabolite production - deletion was shown to increase production of several secondary metabolites but decrease the production of gliotoxin, while conversely a strain overexpressing $h d a A$ had increased gliotoxin biosynthesis [50]. Interestingly, H3K9 methylation levels were slightly higher in $\Delta h d a A$ compared to wild type. Undoubtedly, further work will be necessary to explore the interactions, if any, between histone modification and gliotoxin biosynthesis.

\section{Self-protection against gliotoxin}

The presence of cytochrome $\mathrm{P}_{450}$ monooxygenases and GST involvement in ETP, specifically gliotoxin, biosynthesis is reminiscent of a phase I/II detoxification system (C. Davis, $\mathrm{PhD}$ thesis, National University of Ireland Maynooth, 2011) [26]. Consequently, it is plausible that gliotoxin biosynthesis evolved from an ancestral detoxification system and that gliotoxin, or a biosynthetic intermediate, conferred a significant competitive advantage on the host organism. Indeed, it is now clear $[25,26]$ that $A$. fumigatus sacrifices reducing power (2 GSH) to enable gliotoxin biosynthesis, and it is therefore not inconceivable that enhanced redox homeostasis, antimicrobial defense, or even redox-mediated signaling, as a consequence of gliotoxin biosynthesis - and presence - drove cluster evolution. However, the selective advantage conferred upon ETP-producing organisms came at a price, and self-protection against redox-active molecular species is also an essential characteristic in these species. Specifically, in addition to effecting disulfide bridge closure during gliotoxin biosynthesis, GliT has been shown to confer resistance against exogenous gliotoxin $[10,11]$ (Figure 3). Moreover, gliT expression is induced by gliotoxin independently of gliZ [10], which further underpins its essential role in self-protection. Interestingly, the alternative transcription factor which influences gliZ-independent gliT expression remains elusive.

GliA-mediated gliotoxin efflux from $A$. fumigatus contributes to self-protection [30] (Figures 2 and 3), and SirA, a GliA ortholog in Leptosphaeria maculans, likewise confers resistance to exogenous sirodesmin [51]. A. fumigatus

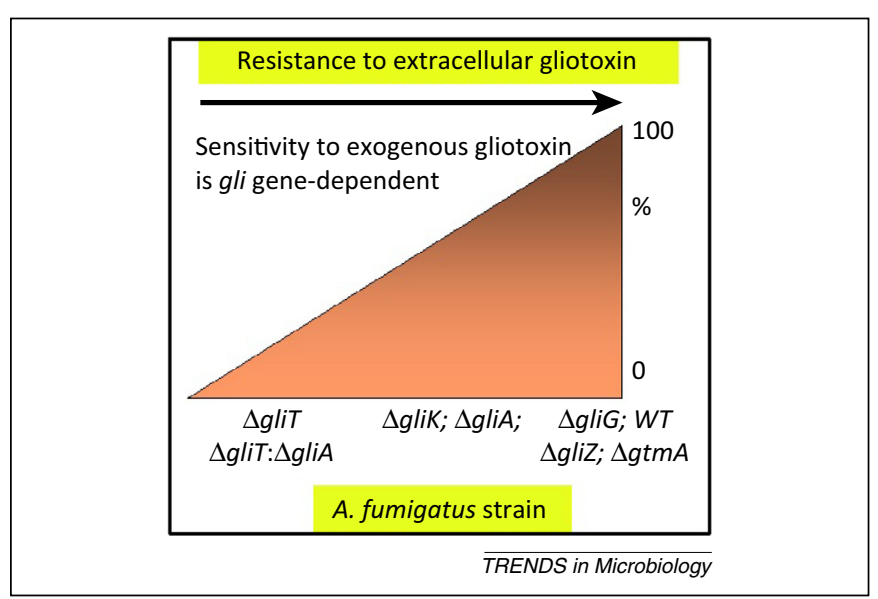

Figure 3. Differential resistance to extracellular gliotoxin following selected $\mathrm{gli}$ cluster gene and gtmA deletion in Aspergillus fumigatus. Combined deletion of gliotoxin oxidoreductase gliT and MFS transporter gliA results in the most gliotoxin-sensitive deletion strain [30], along with $\Delta$ gliT. A. fumigatus wild type (WT), $\Delta$ gliG, $\Delta$ gtmA and $\Delta$ gliZ exhibit intact gliotoxin-resistance, while $\Delta g$ liK and $\Delta$ gliA exhibit intermediate sensitivity. 
$\Delta g l i A: \Delta g l i T$ is also highly sensitive to exogenous gliotoxin (Figure 3), and this led Wang et al. [30] to conclude that they represent independent self-protection systems. However, an alternative hypothesis whereby GliT-mediated gliotoxin oxidation is necessary for GliA-mediated efflux is also compatible with their experimental observations. These authors also observed that attenuated gliotoxin levels are secreted by A. fumigatus $\Delta$ gliA and they posited the existence of an alternative gliotoxin efflux mechanism. However, demonstration of a supplementary secretion system remains outstanding. The identification of bis-thiomethylated holomycin in the bacteria Streptomyces clavuligerus, and its role in deactivation of the dithiol 'warhead', led to speculation about the presence of a back-up selfprotection system catalyzed by gliM/gliN (methyltransferases) encoded within the gli cluster in A. fumigatus (Figure 1) [52]. However, it appears that GtmA is the sole enzyme responsible for BmGT formation in A. fumigatus and that its primary role is the diminution of gliotoxin biosynthesis and not as a back-up self-protection system involving the direct detoxification of dithiol gliotoxin via bis-thiomethylation [8]. Indeed, deletion of gtmA has no significant impact on sensitivity of $A$. fumigatus to exogenous gliotoxin (Figure 3), which implies that GliT is the key mediator of this self-protection system.

\section{The gliotoxin-related metabolome}

Increasingly, examples of fungal secondary metabolite cross-talk, whereby abrogation or overproduction of one secondary metabolite may affect the production of another apparently unrelated metabolite are emerging, as reviewed in [53]. This phenomenon has been described in A. fumigatus in relation to gliotoxin biosynthesis, whereby overexpression of gliZ led to helvolic acid production at $37^{\circ} \mathrm{C}$ which was not observed in wild type, while the production of multiple metabolites was altered by the loss or gain of gliZ, suggesting that gliZ may influence the biosynthesis of secondary metabolites other than gliotoxin [19]. In a later study employing 2D NMR spectroscopy (DANS) which compared metabolite extracts from wild type and $\Delta g l i Z$, nine novel gliZ-dependent metabolites were identified in addition to previously identified metabolites [9] (Figure 1).

Disruption of gliotoxin biosynthesis in A. fumigatus $\Delta g l i K$ prevents the degradation and recycling of $2 \mathrm{~mol}$ GSH per mol gliotoxin produced, and this resulted in the concomitant acquisition of an oxidative stress phenotype accompanied by the first identification of the antioxidant ergothioneine (EGT) in A. fumigatus [17]. Moreover, significantly elevated EGT levels, possibly due to altered redox homeostasis, were evident in A. fumigatus $\Delta$ gliK [17]. The systems significance of the interactions between these three redox-active species, GSH, gliotoxin, and EGT, remains to be elucidated. In addition, significantly increased levels of two unrelated metabolites of $\mathrm{m} / \mathrm{z}$ 394 and 396 were also reported, implying that disruption of gliotoxin biosynthesis, specifically via gli $K$ deletion, influences the production of other, unrelated natural products [17]. Further evidence of this phenomenon was observed in A. fumigatus $\Delta g l i T$ where production of brevianamide $\mathrm{F}$ was significantly increased, while production of tryprostatin A and $\mathrm{B}$, fumagillin, and pseurotin A was significantly decreased [54]. This suggests that although GliT-mediated disulfide bridge closure is required for gliotoxin biosynthesis, abrogation of this redox reaction dysregulates the biosynthesis of other secondary metabolites, raising the question as to whether this is the case in other microbial species with similar disulfide bridge-forming systems (e.g., holomycin in Streptomyces clavuligerus and Yersinia ruckeri) $[55,56]$. In any case, it is now clear that disruption of gliotoxin biosynthesis in A. fumigatus is a trans-consequential event which has far-reaching effects on secondary metabolism, possibly as a result of A. fumigatus compensating for the loss of gliotoxin-influenced redox homeostasis.

\section{Gliotoxin functionality in fungi}

With the discovery of GliT as the key to A. fumigatus selfprotection against gliotoxin toxicity $[10,11]$, and the demonstration of how gliotoxin can inhibit growth of Aspergilli and pathogenic fungi such as Candida albicans [57,92], the intriguing questions of the true in vivo role(s) of this molecule and the in vivo effects upon fungi, either gliotoxin producers or not, was brought to the fore. While the exact mode of action of gliotoxin in vivo remains to be fully elucidated, studies in yeast, Aspergillus spp., and mammalian cell lines have demonstrated that elevated intracellular levels of GSH can exacerbate toxicity [15,58,59], effectively by reducing the gliotoxin disulfide bridge to yield reactive dithiol gliotoxin and thus allowing many as-yet unidentified detrimental interactions to occur between reduced gliotoxin and essential cellular components. The importance of elevated GSH levels in mediating gliotoxin toxicity in fungi is particularly highlighted by the ability of yeast deleted for gsh1 (the gene encoding $\gamma$ glutamylcysteine synthetase which is essential for GSH biosynthesis) to grow much better than wild type in the presence of high levels of exogenous gliotoxin, and by the elevated GSH levels in gliotoxin-sensitive A. fumigatus $\Delta g l i T[15]$.

In an attempt to gain insight into the in vivo effects of gliotoxin exposure, Chamilos and colleagues [60] screened a yeast deletion library to identify strains that were sensitive or resistant to exogenous gliotoxin compared to wild type. While a number of gene deletions caused changes in the gliotoxin exposure profile, of particular note was the finding that deletion of cys 3 , which encodes a cystathionine $\gamma$-lyase, caused sensitization to gliotoxin. Cys3 is the second enzyme in the reverse transulfuration pathway and is responsible for converting homocysteine, a product of $S$ adenosylmethionine metabolism, to cysteine [61]. Given recent studies demonstrating the importance of GSH in mediating gliotoxin sensitivity [15,17], and a recent global transcriptome study demonstrating major alterations in sulfur metabolism genes in A. fumigatus $\Delta g l i T$ upon gliotoxin exposure [54], the finding that cys3 deletion sensitizes yeast to gliotoxin alludes to evolutionarily conserved in vivo molecular responses to gliotoxin exposure and perhaps toxicity.

The exposure of mammalian cells to gliotoxin increases the levels of reactive oxygen species (ROS) [62], and deletion of yeast genes responsible for dealing with oxidative stress causes cells to be sensitized to gliotoxin [15]. 
However, the relationship between gliotoxin and oxidative stress induction is complicated. In addition to the aforementioned relationship between GSH and gliotoxin, recent studies in fungi have demonstrated that the presence of gliotoxin can increase survival and growth when A. fumigatus is cultured in the presence of high levels of growthinhibiting $\mathrm{H}_{2} \mathrm{O}_{2}$ [17]. Moreover, gliotoxin can directly reduce the levels of ROS produced in A. fumigatus when exposed to $\mathrm{H}_{2} \mathrm{O}_{2}$, suggesting that under appropriate conditions gliotoxin can exert antioxidant activities [63]. While the mechanism of such activity remains to be elucidated, it is clear from proteomic studies that gliotoxin exposure, either alone or in combination with $\mathrm{H}_{2} \mathrm{O}_{2}$, can have farreaching effects on a variety of central metabolic processes such as amino acid and purine metabolism in fungi [63]. It is not inconceivable that these global changes in the fungal proteome each provide a systems-level contribution to alleviating oxidative stress induced by $\mathrm{H}_{2} \mathrm{O}_{2}$ exposure.

\section{Gliotoxin impact on animal cells}

Gliotoxin and related ETPs directly inactivate multiple enzymes and proteins in animal cells, including nuclear factor-кB (NF-кB), NADPH oxidase, and glutaredoxin, by conjugation to thiol groups [64-66]. It has been conclusively demonstrated that gliotoxin is taken up by animal cells in a GSH-dependent manner whereby this normally protective molecular species facilitates the concentration of gliotoxin within animal cells [59]. Specifically, upon uptake intracellular gliotoxin is reduced to the dithiol form, resulting in GSH depletion and concomitant formation of GSSG. Apoptosis ensues, followed by oxidation of dithiol gliotoxin to reform gliotoxin [59]. Gliotoxin then effluxes from the cell in a pseudocatalytic manner and may be taken up by adjacent cells to continue its cytocidal effects. In addition to having an essential role in gliotoxin biosynthesis as a sulfur donor $[25,26]$, GSH has also been shown to have an important role in mediating gliotoxin-induced cytotoxicity in animal cells and fungi. Interestingly, diminution of intracellular GSH levels using L-buthionine-sulfoxamine (BSO), a specific inhibitor of GSH synthesis, significantly attenuated the cytotoxic effect of gliotoxin towards human neuroblastoma SHSY5Y cells, possibly due to a deficit in GSH-mediated cleavage of the disulfide bridge of gliotoxin to produce reactive dithiol gliotoxin [58]. This study highlighted the seemingly contradictory effects of antioxidants in enhancing cellular sensitivity to disulfide compounds.

Peroxiredoxins (Prxs) constitute a ubiquitous family of antioxidant enzymes that catalyze the reduction of peroxides. This is achieved with two electrons derived from NADPH via an electron-conveying system comprising thioredoxin and thioredoxin reductase. Nanomolar levels of gliotoxin can protect against oxidative damage by converting $\mathrm{H}_{2} \mathrm{O}_{2}$ to water in HeLa cells, where it functions to accept electrons from NADPH in the thioredoxin redox system [6]. Gliotoxin could, in turn, attenuate $\mathrm{H}_{2} \mathrm{O}_{2}$-induced angiogenesis in a dose-dependent manner, thereby acting as an anti-angiogenic agent. This anti-angiogenic effect of A. fumigatus was completely attenuated by deletion of laeA, the global regulator of secondary metabolism [67]. These studies also revealed that gliotoxin was responsible for approximately $50 \%$ of this anti-angiogenic activity because culture extracts from A. fumigatus $\Delta g l i P$, deficient in gliotoxin biosynthesis only, did not abolish angiogenesis. In murine melanoma cells, Prx II silencing enhanced lung metastasis in vivo. Gliotoxin, owing to its ability to exert a Prx-like activity, inhibited the proliferation and migration as well as lung metastasis of Prx II-deficient melanoma cells. This in vivo study implicated the Prx II mimetic ability of gliotoxin as a promising therapeutic drug for preventing melanoma metastasis [68].

Further studies have dissected the molecular basis and investigated applications of ETP-mediated anti-angiogenesis. Tumor cells must adapt to a hypoxic environment to survive and grow. This is mediated by hypoxia-inducible factor (HIF-1), whereby the $\mathrm{O}_{2}$-regulated HIF- $1 \alpha$ subunit of the HIF-1 transcription factor is required for activation of multiple genes involved in tumor progression and angiogenesis [69]. Under hypoxic conditions, HIF-1 $\alpha$ undergoes nuclear translocation where it interacts with HIF-1 $\beta$ and binds to target DNA sequences. Upon recruitment of additional proteins (e.g., p300), gene expression is activated to mount an adaptive response to hypoxia [70]. It has been speculated that disruption of this hypoxic response could form an effective anticancer strategy. Interestingly, gliotoxin and the ETPs chaetocin and chetomin can inhibit the HIF-1 $\alpha-$ p300 interaction by ejecting an essential $\mathrm{Zn}^{2+}$ cofactor from p300 via the formation of a $\mathrm{Zn}^{2+}(\mathrm{ETP})_{2}$ moiety [71]. Moreover, exposure to gliotoxin resulted in anti-proliferative effects on cultured tumor cells, which were reversible by $\mathrm{Zn}^{2+}$ supplementation. Furthermore, $\mathrm{Zn}^{2+}$ can protect HepG2 cells against toxicity of the ETP sporidesmin from Pithomyces chartarum which causes facial eczema in sheep [72]. Indeed, $\mathrm{Zn}^{2+}$ supplementation of ruminant diets is an effective prophylactic treatment for this disease [73]; it is tempting to speculate that this could be effected via disruption of the HIF- $1 \alpha-p 300$ adaptive response. More recently it has been shown that gliotoxin, chaetocin, and chetomin could significantly decrease tumor growth in a prostate cancer xenograft model system [69]. Although gliotoxin exposure did not impact on the expression of HIF- $1 \alpha$ regulated genes, these authors speculated that the antitumor activity of gliotoxin could be mediated via farnesyltransferase inhibition because inhibition of this enzyme can attenuate angiogenesis by interfering with endothelial cell migration [74].

As described in Figure 4, gliotoxin induces apoptotic cell death by activating the pore-forming proapoptotic Bcl-2 family member Bak to elicit ROS generation, the mitochondrial release of apoptogenic factors, and caspase-3 activation. Thus, not only has this work elucidated the mechanism of gliotoxin-induced apoptosis but also it has uncovered the molecular mechanisms of Bcl-2 family initiation $[16,75]$. It is possible that induction of gliotoxindependent apoptosis could impact on the severity and course of Aspergillus infection by destroying lung epithelium or by killing alveolar macrophages [62]. NF- $\mathrm{kB}$ is a ubiquitously expressed proinflammatory transcription factor composed of different combinations of members of the Rel family of proteins. As shown in Figure 4 gliotoxin has been well-characterized as an inhibitor of NF- $\mathrm{KB}$ activation [64], and this then led to a plethora of studies which utilize gliotoxin as a specific NF-кB inhibitor [76-78]. Gliotoxin 


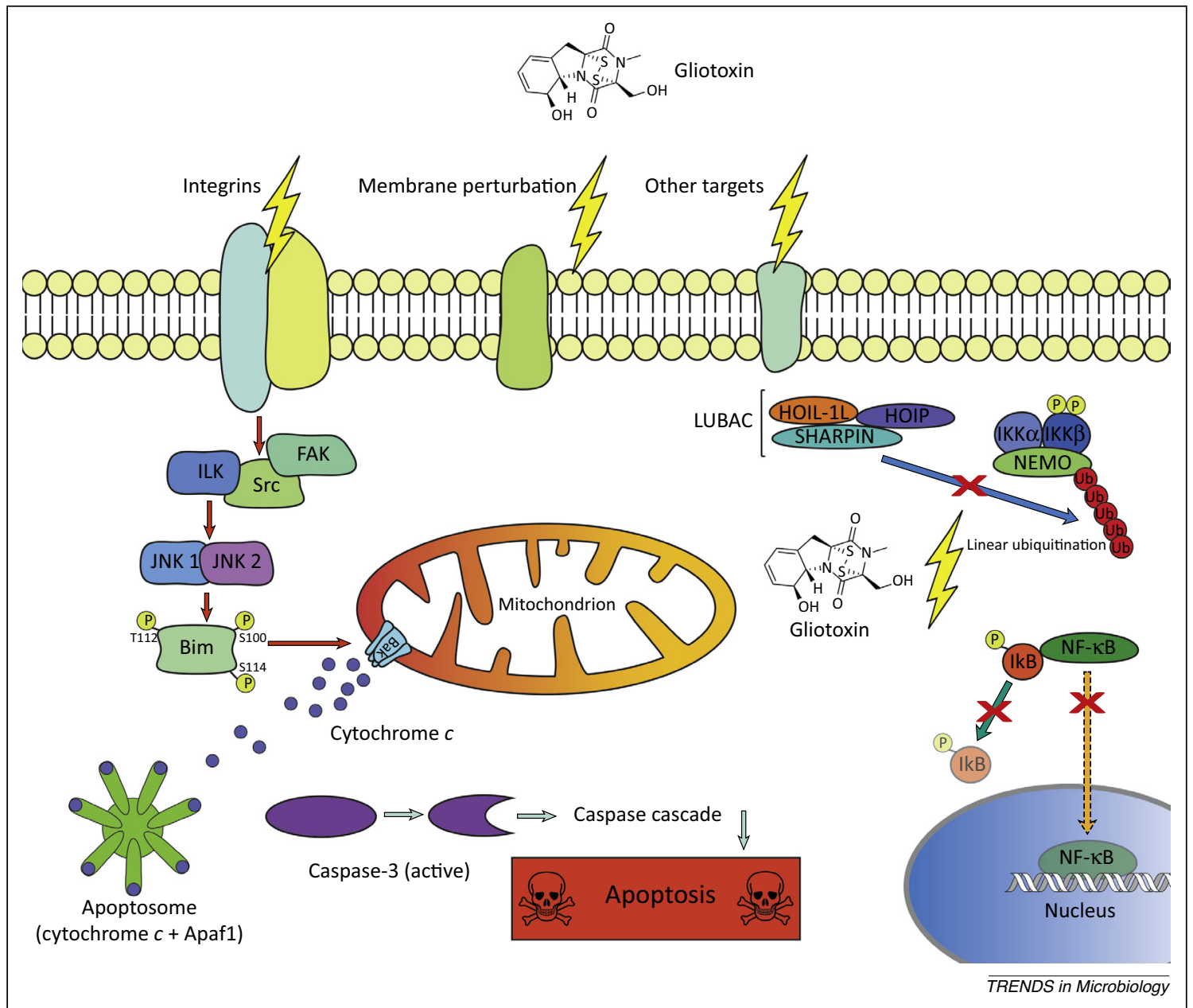

Figure 4. Gliotoxin induction of apoptosis in mammalian cells through various specific cellular targets. Upstream of the pore-forming proapoptotic Bcl-2 family member Bak, gliotoxin-mediated apoptosis requires the c-Jun N-terminal kinase 1 and 2 (JNK1/2)-mediated phosphorylation (P) of the BH3-only protein BimEL at three sites (S100, T112, and $\mathrm{S} 114)$. This triple phosphorylation increases the stability of BimEL, increases its binding affinity for Bcl-2-like survival factors, and activates Bak more effectively. Notably, triplemutated Bim is unable to transduce the gliotoxin-JNK-initiated apoptotic stimulus to Bak. Compared to wild type, mice lacking Bak were significantly less susceptible to $A$. fumigatus infection, which demonstrates the in vivo relevance of this gliotoxin-induced apoptotic pathway $[16,62,75]$. In resting cells, NF- $\mathrm{B}$ is localized to the cytoplasm because of binding to inhibitory protein IkB. Upon activation, NF-KB inducing kinase (NIK) is activated, which in turn activates a complex of specific IkB kinases (IKKs) resulting in IkB phosphorylation. Phosphorylation of IkB leads to a rapid ubiquitination (Ub) which makes it a substrate for the proteasome. The active NF-kB complex then translocates to the nucleus and initiates the expression of target genes. Gliotoxin inhibits NF-кB activation by preventing IkB degradation [64]. Gliotoxin also suppresses NF-kB activation by inhibiting the linear ubiquitin (Ub) chain assembly complex (LUBAC). This is the first selective small-molecule inhibitor of this complex to be identified [91]. Abbreviations: Apaf1, apoptotic protease activating factor 1; FAK, focal adhesion kinase; HOIL-1L, heme-oxidized IRP2 ubiquitin ligase-1; HOIP, HOIL-1L interacting protein; ILK, integrin-linked kinase; NEMO, NF-кB essential modulator; SHARPIN, SHANK-associated RH domain interacting protein; Src, Sarcoma family nonreceptor protein tyrosine kinase.

has also been utilized to demonstrate that NF-кB inhibition reverses tumor necrosis factor- $\alpha(\mathrm{TNF}-\alpha)$-induced eosinophil survival, highlighting the major role of $\mathrm{NF}-\kappa \mathrm{B}$ in TNF- $\alpha$-induced inhibition of eosinophil apoptosis [79]. It is clear from these selected studies that gliotoxin is a potent small molecule capable of targeting and revealing multiple aspects of animal cell biochemistry.

\section{Concluding remarks}

Our knowledge of gliotoxin biosynthesis, functionality, and utility has increased dramatically over recent years [80]. Specifically, it is now clear that gliotoxin biosynthesis (i) involves novel enzymology, (ii) unusually deploys GSH as a substrate for biosynthetic intermediate sulfurization, (iii) has resulted in the evolution of a self-protection system mediated by GliT, (iv) results in the production of a suite of additional related metabolites (some with diagnostic potential $[38,81])$, and (v) is post-biosynthetically negatively regulated by GtmA activity. Reconsidering the established view of gliotoxin as a 'toxin', it is becoming increasingly clear that its antioxidant and signaling roles [82] need further evaluation and study. Whether this antioxidant ability is related to the influence that gliotoxin, or pathway components, exert on the biosynthesis of unrelated metabolites in A. fumigatus remains to be established (Box 1). GSH-mediated reduction of gliotoxin in animal cells and

\section{Box 1. Outstanding questions}

- What are the roles of gliH and gliM in gliotoxin biosynthesis?

- What are the interactions and roles of the many regulatory genes which appear to control gliotoxin biosynthesis?

- What is the mechanism of independent regulation of gliT expression?

- How can the interplay between gliotoxin and other natural product biosynthetic pathways in A. fumigatus be elucidated?

- What is the redox systems significance of the interplay between GSH, EGT, and gliotoxin in fungi?

- Is there an anticancer role of gliotoxin and related ETPs? Can its role as a probe of cellular systems in animal cells be further developed? 
fungi appears to be central to the potency of the metabolite to alter and impair cellular systems. In the future, contextualization of gliotoxin effects on eukaryotic cells may benefit from cognizance of its multiple actions and reactivity of dithiol gliotoxin. Moreover, in fungi capable of gliotoxin biosynthesis, we now know that exogenous gliotoxin induces gli cluster expression and concomitant gliotoxin biosynthesis [21,54]; the impacts and utility of these observations cannot be underestimated.

\section{Acknowledgments}

Gliotoxin research in the laboratory of S.D. is funded by a Science Foundation Ireland Principal Investigator Award (SFI/11/PI/1188). S.K.D. is an Irish Research Council Postgraduate Scholar.

\section{References}

1 Kwon-Chung, K. and Sugui, J. (2013) Aspergillus fumigatus - what makes the species a ubiquitous human fungal pathogen? PLoS Pathog. 9, e1003743

2 Shi, L. et al. (2012) Immunoproteomics based identification of thioredoxin reductase GliT and novel Aspergillus fumigatus antigens for serologic diagnosis of invasive aspergillosis. BMC Microbiol. 12, 11

3 Agarwal, R. et al. (2013) Allergic bronchopulmonary aspergillosis: review of literature and proposal of new diagnostic and classification criteria. Clin. Exp. Allergy 43, 850-873

4 Haas, H. (2014) Fungal siderophore metabolism with a focus on Aspergillus fumigatus. Nat. Prod. Rep. 31, 1266-1276

5 Sugui, J.a. et al. (2007) Gliotoxin is a virulence factor of Aspergillus fumigatus: gliP deletion attenuates virulence in mice immunosuppressed with hydrocortisone. Eukaryot. Cell 6, 1562-1569

6 Choi, H. et al. (2007) Discovery of gliotoxin as a new small molecule targeting thioredoxin redox system. Biochem. Biophys. Res. Commun. $359,523-528$

7 Chai, C. and Waring, P. (2000) Redox sensitive epidithiodioxopiperazines in biological mechanisms of toxicity. Redox Rep. 5, 257-264

8 Dolan, S.K. et al. (2014) Regulation of nonribosomal peptide synthesis: bis-thiomethylation attenuates gliotoxin biosynthesis in Aspergillus fumigatus. Chem. Biol. 21, 999-1012

9 Forseth, R.R. et al. (2011) Identification of cryptic products of the gliotoxin gene cluster using NMR-based comparative metabolomics and a model for gliotoxin biosynthesis. J. Am. Chem. Soc. 133, 96789681

10 Schrettl, M. et al. (2010) Self-protection against gliotoxin - a component of the gliotoxin biosynthetic cluster, GliT, completely protects Aspergillus fumigatus against exogenous. PLoS Pathog. 6 , e1000952

11 Scharf, D.H. et al. (2010) Transannular disulfide formation in gliotoxin biosynthesis and its role in self-resistance of the human pathogen Aspergillus fumigatus. J. Am. Chem. Soc. 132, 10136-10141

12 Patron, N.J. et al. (2007) Origin and distribution of epipolythiodioxopiperazine (ETP) gene clusters in filamentous ascomycetes. BMC Evol. Biol. 7, 174

13 Welch, T.R. and Williams, R.M. (2014) Epidithiodioxopiperazines. occurrence, synthesis and biogenesis. Nat. Prod. Rep. 31, 1376-1404

14 Cherblanc, F.L. et al. (2013) Mechanistic and chiroptical studies on the desulfurization of epidithiodioxopiperazines reveal universal retention of configuration at the bridgehead carbon atoms. J. Org. Chem. 78, 11646-11655

15 Carberry, S. et al. (2012) Gliotoxin effects on fungal growth: mechanisms and exploitation. Fungal Genet. Biol. 49, 302-312

16 Häcker, G. (2013) A Bim-activating mould. Cell Death Differ. 20, 12891290

17 Gallagher, L. et al. (2012) The Aspergillus fumigatus protein GliK protects against oxidative stress and is essential for gliotoxin biosynthesis. Eukaryot. Cell 11, 1226-1238

18 Gardiner, D.M. and Howlett, B.J. (2005) Bioinformatic and expression analysis of the putative gliotoxin biosynthetic gene cluster of Aspergillus fumigatus. FEMS Microbiol. Lett. 248, 241-248

19 Bok, J.W. et al. (2006) GliZ, a transcriptional regulator of gliotoxin biosynthesis, contributes to Aspergillus fumigatus virulence. Infect. Immun. 74, 6761-6768
20 Balibar, C.J. and Walsh, C.T. (2006) GliP, a multimodular nonribosomal peptide synthetase in Aspergillus fumigatus, makes the diketopiperazine scaffold of gliotoxin. Biochemistry 45, 1502915038

21 Cramer, R.A. et al. (2006) Disruption of a nonribosomal peptide synthetase in Aspergillus fumigatus eliminates gliotoxin production. Eukaryot. Cell 5, 972-980

22 Kupfahl, C. et al. (2006) Deletion of the gliP gene of Aspergillus fumigatus results in loss of gliotoxin production but has no effect on virulence of the fungus in a low-dose mouse infection model. Mol. Microbiol. 62, 292-302

23 Spikes, S. and Xu, R. (2008) Gliotoxin production in Aspergillus fumigatus contributes to host-specific differences in virulence. $J$. Infect. Dis. 197, 479-486

24 Chang, S-L. et al. (2013) Reconstitution of the early steps of gliotoxin biosynthesis in Aspergillus nidulans reveals the role of the monooxygenase GliC. Bioorg. Med. Chem. Lett. 23, 2155-2157

25 Davis, C. et al. (2011) The role of glutathione S-transferase GliG in gliotoxin biosynthesis in Aspergillus fumigatus. Chem. Biol. 18, $542-552$

26 Scharf, D.H. et al. (2011) A dedicated glutathione S-transferase mediates carbon-sulfur bond formation in gliotoxin biosynthesis. $J$. Am. Chem. Soc. 133, 12322-12325

27 Scharf, D.H. et al. (2013) Epidithiodiketopiperazine biosynthesis: a four-enzyme cascade converts glutathione conjugates into transannular disulfide bridges. Angew. Chem. Int. Ed. Engl. 52, 11092-11095

28 Scharf, D.H. et al. (2012) Epidithiol formation by an unprecedented twin carbon-sulfur lyase in the gliotoxin pathway. Angew. Chem. Int. Ed. Engl. 124, 10211-10215

29 Scharf, D.H. et al. (2014) Opposed effects of enzymatic gliotoxin $\mathrm{N}$-and S-methylations. J. Am. Chem. Soc. 136, 11674-11679

30 Wang, D. et al. (2014) GliA in Aspergillus fumigatus is required for its tolerance to gliotoxin and affects the amount of extracellular and intracellular gliotoxin. Med. Mycol. 52, 506-518

31 Schoberle, T.J. et al. (2014) A novel C2H2 transcription factor that regulates gliA expression interdependently with GliZ in Aspergillus fumigatus. PLoS Genet. 10, e1004336

32 Perrin, R.M. et al. (2007) Transcriptional regulation of chemical diversity in Aspergillus fumigatus by LaeA. PLoS Pathog. 3, e50

33 Bayram, O. et al. (2008) VelB/VeA/LaeA complex coordinates light signal with fungal development and secondary metabolism. Science 320, 1504-1506

34 Bok, J.W. and Keller, N.P. (2004) LaeA, a regulator of secondary metabolism in Aspergillus spp. Eukaryot. Cell 8, 527-535

$35 \mathrm{Bok}$, J. et al. (2005) LaeA, a regulator of morphogenetic fungal virulence factors. Eukaryot. Cell 4, 1574-1582

36 Dhingra, S. et al. (2012) VeA regulates conidiation, gliotoxin production, and protease activity in the opportunistic human pathogen Aspergillus fumigatus. Eukaryot. Cell 11, 1531-1543

37 Smith, T.D. and Calvo, A.M. (2014) The $m t f A$ transcription factor gene controls morphogenesis, gliotoxin production, and virulence in the opportunistic human pathogen Aspergillus fumigatus. Eukaryot. Cell $13,766-775$

38 Sekonyela, R. et al. (2013) RsmA regulates Aspergillus fumigatus gliotoxin cluster metabolites including cyclo(L-Phe-L-Ser), a potential new diagnostic marker for invasive aspergillosis. PLoS ONE 8, e62591

39 Gravelat, F.N. et al. (2008) In vivo analysis of Aspergillus fumigatus developmental gene expression determined by real-time reverse transcription-PCR. Infect. Immun. 76, 3632-3639

40 Twumasi-Boateng, K. et al. (2009) Transcriptional profiling identifies a role for BrlA in the response to nitrogen depletion and for StuA in the regulation of secondary metabolite clusters in Aspergillus fumigatus. Eukaryot. Cell 8, 104-115

$41 \mathrm{Kim}, \mathrm{S}$. and Shin, K. (2013) The developmental regulators, FlbB and FlbE, are involved in the virulence of Aspergillus fumigatus. J. Microbiol. Biotechnol. 23, 766-770

42 Xiao, P. et al. (2010) Aspergillus fumigatus flbB encodes two basic leucine zipper domain (bZIP) proteins required for proper asexual development and gliotoxin production. Eukaryot. Cell 9, 1711-1723

43 Kwon, N-J. et al. (2010) Characterization of the developmental regulator FlbE in Aspergillus fumigatus and Aspergillus nidulans. Fungal Genet. Biol. 47, 981-993 
44 Palmer, J.M. et al. (2013) Loss of CclA, required for histone 3 lysine 4 methylation, decreases growth but increases secondary metabolite production in Aspergillus fumigatus. PeerJ 1, e4

45 Takahashi, M. et al. (2012) Inhibition of histone H3K9 methyltransferases by gliotoxin and related epipolythiodioxopiperazines. J. Antibiot. 65, 263-265

46 Cherblanc, F.L. et al. (2013) On the histone lysine methyltransferase activity of fungal metabolite chaetocin. J. Med. Chem. 56, 8616-8625

47 Strauss, J. and Reyes-Dominguez, Y. (2011) Regulation of secondary metabolism by chromatin structure and epigenetic codes. Fungal Genet. Biol. 48, 62-69

$48 \mathrm{Bok}$, J. and Chiang, Y. (2009) Chromatin-level regulation of biosynthetic gene clusters. Nat. Chem. Biol. 5, 462-464

49 Shwab, E.K. et al. (2007) Histone deacetylase activity regulates chemical diversity in Aspergillus. Eukaryot. Cell 6, 1656-1664

50 Lee, I. et al. (2009) HdaA, a class 2 histone deacetylase of Aspergillus fumigatus, affects germination and secondary metabolite production. Fungal Genet. Biol. 46, 782-790

51 Gardiner, D.M. et al. (2005) The ABC transporter gene in the sirodesmin biosynthetic gene cluster of Leptosphaeria maculans is not essential for sirodesmin production but facilitates selfprotection. Fungal Genet. Biol. 42, 257-263

52 Li, B. et al. (2012) A backup plan for self-protection: S-methylation of holomycin biosynthetic intermediates in Streptomyces clavuligerus. Chembiochem 13, 2521-2526

53 Sheridan, K.J. et al. (2015) Endogenous cross-talk of fungal metabolites. Front. Microbiol. 5, 10.3389

54 O'Keeffe, G. et al. (2014) RNA-seq reveals the pan-transcriptomic impact of attenuating the gliotoxin self-protection mechanism in Aspergillus fumigatus. BMC Genomics 15, 894

55 Qin, Z. et al. (2013) The fish pathogen Yersinia ruckeri produces holomycin and uses an RNA methyltransferase for self-resistance. $J$. Biol. Chem. 288, 14688-14697

$56 \mathrm{Li}$, B. and Walsh, C. (2011) Streptomyces clavuligerus HlmI is an intramolecular disulfide-forming dithiol oxidase in holomycin biosynthesis. Biochemistry 50, 4615-4622

57 Losada, L. et al. (2009) Effect of competition on the production and activity of secondary metabolites in Aspergillus species. Med. Mycol. 47 (Suppl 1), S88-S96

58 Axelsson, V. et al. (2006) Glutathione intensifies gliotoxin-induced cytotoxicity in human neuroblastoma SH-SY5Y cells. Cell Biol. Toxicol. 22, 127-136

59 Bernardo, P.H. et al. (2003) A novel redox mechanism for the glutathione-dependent reversible uptake of a fungal toxin in cells. $J$. Biol. Chem. 278, 46549-46555

60 Chamilos, G. et al. (2008) Genomewide screening for genes associated with gliotoxin resistance and sensitivity in Saccharomyces cerevisiae. Antimicrob. Agents Chemother. 52, 1325-1329

61 Hopwood, E.M. et al. (2014) A role for glutamate-333 of Saccharomyces cerevisiae cystathionine $\gamma$-lyase as a determinant of specificity. Biochim. Biophys. Acta 1844, 465-472

62 Pardo, J. et al. (2006) The mitochondrial protein Bak is pivotal for gliotoxin-induced apoptosis and a critical host factor of Aspergillus fumigatus virulence in mice. J. Cell Biol. 174, 509-519

63 Owens, R. et al. (2014) A proteomic approach to investigating gene cluster expression and secondary metabolite functionality in Aspergillus fumigatus. PLoS ONE 9, e106942

64 Pahl, H.L. et al. (1996) The immunosuppressive fungal metabolite gliotoxin specifically inhibits transcription factor NF-kappaB. J. Exp. Med. 183, 1829-1840

65 Tsunawaki, S. et al. (2004) Fungal metabolite gliotoxin inhibits assembly of the human respiratory burst NADPH oxidase. Infect. Immun. 72, 3373-3382

66 Srinivasan, U. et al. (2006) Selective inactivation of glutaredoxin by sporidesmin and other epidithiopiperazinediones. Biochemistry 45, 8978-8987

67 Ben-Ami, R. et al. (2009) Aspergillus fumigatus inhibits angiogenesis through the production of gliotoxin and other secondary metabolites. Blood 114, 5393-5399

68 Lee, D.J. et al. (2013) Peroxiredoxin-2 represses melanoma metastasis by increasing E-cadherin/ $\alpha$-catenin complexes in adherens junctions. Cancer Res. 73, 4744-4757
69 Reece, K.M. et al. (2014) Epidithiodiketopiperazines (ETPs) exhibit in vitro antiangiogenic and in vivo antitumor activity by disrupting the HIF-1 $1 \alpha /$ p300 complex in a preclinical model of prostate cancer. Mol. Cancer 13, 91

70 Palazon, A. et al. (2014) HIF transcription factors, inflammation, and immunity. Immunity 41, 518-528

71 Cook, K.M. et al. (2009) Epidithiodiketopiperazines block the interaction between hypoxia-inducible factor-1alpha (HIF-1alpha) and p300 by a zinc ejection mechanism. J. Biol. Chem. 284, 2683126838

72 Duncan, E.J. et al. (2005) Zinc protection of HepG2 cells from sporidesmin toxicity does not require de novo gene transcription. Toxicol. Lett. 159, 164-172

73 Munday, R. et al. (2001) A zinc-containing intraruminal device for prevention of the sporidesmin-induced cholangiopathy of facial eczema in calves. N. Z. Vet. J. 40, 29-33

74 Peng, G. et al. (2012) Inhibition of farnesyltransferase reduces angiogenesis by interrupting endothelial cell migration. Biochem. Pharmacol. 83, 1374-1382

75 Geissler, A. et al. (2013) Apoptosis induced by the fungal pathogen gliotoxin requires a triple phosphorylation of Bim by JNK. Cell Death Differ. 20, 1317-1329

76 Fitzpatrick, L.R. et al. (2002) Gliotoxin, an inhibitor of nuclear factorkappa B, attenuates peptidoglycan-polysaccharide-induced colitis in rats. Inflamm. Bowel Dis. 8, 159-167

77 Herfarth, H. et al. (2000) Nuclear factor-kappa B activity and intestinal inflammation in dextran sulphate sodium (DSS)-induced colitis in mice is suppressed by gliotoxin. Clin. Exp. Immunol. 120, 59-65

78 Ward, C. et al. (1999) NF-kappa B activation is a critical regulator of human granulocyte apoptosis in vitro. J. Biol. Chem. 274, 43094318

79 Kankaanranta, H. et al. (2014) Tumour necrosis factor- $\alpha$ regulates human eosinophil apoptosis via ligation of TNF-receptor 1 and balance between NF-кB and AP-1. PLoS ONE 9, е90298

80 Scharf, D.H. et al. (2012) Biosynthesis and function of gliotoxin in Aspergillus fumigatus. Appl. Microbiol. Biotechnol. 93, 467-472

81 Domingo, M.P. et al. (2012) Bis(methyl)gliotoxin proves to be a more stable and reliable marker for invasive aspergillosis than gliotoxin and suitable for use in diagnosis. Diagn. Microbiol. Infect. Dis. 73, $57-64$

82 Zheng, H. et al. (2014) Redox metabolites signal polymicrobial biofilm development via the NapA oxidative stress cascade in Aspergillus. Curr. Biol. 25, 29-37

83 Scharf, D.H. et al. (2014) Flavoenzyme-catalyzed formation of disulfide bonds in natural products. Angew. Chem. Int. Ed. Engl. $53,2221-2224$

84 Dhingra, S. et al. (2013) The fumagillin gene cluster, an example of hundreds of genes under veA control in Aspergillus fumigatus. PLoS ONE 8, e77147

85 Park, H-S. et al. (2012) Characterization of the velvet regulators in Aspergillus fumigatus. Mol. Microbiol. 86, 937-953

86 Shin, K-S. et al. (2013) Comparative proteomic analyses reveal that FlbA down-regulates gliT expression and SOD activity in Aspergillus fumigatus. J. Proteomics 87, 40-52

$87 \mathrm{Mah}, \mathrm{J}-\mathrm{H}$. and Yu, J-H. (2006) Upstream and downstream regulation of asexual development in Aspergillus fumigatus. Eukaryot. Cell 5, 15851595

88 Valiante, V. et al. (2008) The mitogen-activated protein kinase MpkA of Aspergillus fumigatus regulates cell wall signaling and oxidative stress response. Fungal Genet. Biol. 45, 618-627

89 Jain, R. et al. (2011) The MAP kinase MpkA controls cell wall integrity, oxidative stress response, gliotoxin production and iron adaptation in Aspergillus fumigatus. Mol. Microbiol. 82, 39-53

90 Kim, S-S. et al. (2013) The developmental regulators, FlbB and FlbE, are involved in the virulence of Aspergillus fumigatus. J. Microbiol. Biotechnol. 23, 766-770

91 Sakamoto, H. et al. (2014) Gliotoxin suppresses NF-кB activation by selectively inhibiting linear ubiquitin chain assembly complex (LUBAC). ACS Chem. Biol. Published online December 17, 2014. http://dx.doi.org/10.1021/cb500653y

92 Coleman, J.J. et al. (2011) Antifungal activity of microbial secondary metabolites. PLoS ONE 6, e25321 